\title{
Magnetisation transfer ratios and transverse magnetisation decay curves in optic neuritis: correlation with clinical findings and electrophysiology
}

J W Thorpe, G J Barker, S J Jones, I Moseley, N Losseff, D G MacManus, S Webb, C Mortimer, D L Plummer, P S Tofts, W I McDonald, D H Miller

\begin{abstract}
Conventional MRI sequences do not permit the distinction between the different pathological characteristics (oedema, demyelination, gliosis, axonal loss) of the multiple sclerosis plaque. Magnetisation transfer imaging and transverse magnetisation decay curve (tMDC) analysis may be more specific. These techniques have been applied to the optic nerves in 20 patients with optic neuritis and the results correlated with clinical and visual evoked potential (VEP) findings. tMDC analysis failed to identify separate intracellular and extracellular water compartments within the optic nerve but gave a measure of transverse relaxation time (T2) without the confounding effects of CSF in the nerve sheath. Both T2 and magnetisation transfer ratio (MTR) were abnormal after an episode of optic neuritis. T2 did not correlate with visual function or with VEP latency or amplitude. There was a significant correlation between MTR reduction and prolongation of VEP latency: this increased latency may reflect an effect of myelin loss on MTR. Longer lesions were associated with worse visual outcome, implying that the overall extent of pathological involvement is likely to influence the degree of functional deficit.
\end{abstract}

(F Neurol Neurosurg Psychiatry 1995;59:487-492)

Keywords: optic neuritis; magnetic resonance imaging; visual evoked potentials

Magnetic resonance imaging often detects clinically silent brain lesions in multiple sclerosis $^{1-7}$ and is increasingly being used to assess the effects of experimental treatments. ${ }^{8-10} \mathrm{~A}$ consistent relation between the extent of MRI abnormalities and disability has not, however, been found. ${ }^{11-14}$ This discrepancy is probably due to several factors (reviewed by McDonald et $a l^{15}$ ) including the lack of pathophysiological specificity of changes seen on conventional proton density and T2 weighted images. The most plausible pathological substrates of functional deficits in multiple sclerosis are demyelination and axonal loss. Demyelination alone, however, is compatible with preserved or even normal function. ${ }^{16-19}$ In experimental models, demyelination can result in complete conduction block, impairment of transmission of impulses at high frequencies, or slowing of conduction $^{20}$; whereas the first will have functional consequences in vivo, the last two may not. Lesions are more likely, therefore, to cause irreversible clinical deficit when there is neuronal loss. Myelin is not visible on conventional MRI and axonal loss by itself may cause no discernible changes. There is some evidence that two quantitative MRI techniques may provide improved pathological specificity:

Transverse magnetisation decay curves The transverse magnetisation decay curve (tMDC) describes the process of transverse (T2) relaxation of magnetisation (of hydrogen protons) after excitation by a radiofrequency pulse. tMDCs can be measured using multiecho spin echo pulse sequences, in which each echo provides one point on the curve. For a homogeneous tissue, most protons have the same T2 and the transverse magnetisation decays as a monoexponential function. Some chronic multiple sclerosis plaques show biexponential tMDCs, suggesting the presence of two distinct proton pools, ${ }^{21-23}$ which may represent intracellular and extracellular water. ${ }^{21} 22$ The presence of biexponential tMDCs is suggestive of an expanded extracellular space, which in brain lesions in chronic multiple sclerosis may be found in the presence of pronounced axonal loss. ${ }^{22}$

Magnetisation transfer imaging

Contrast in conventional MRI mainly results from differences in relaxation times and proton density of mobile (free) water protons; however, there also exists a pool of water protons tightly bound to macromolecules such as proteins and lipid membranes, which, by virtue of their very short relaxation times, are essentially invisible on MRI. By exploiting differences in the resonant properties of the free and bound protons, it is possible to saturate selectively the bound pool. As bound and free pools are in rapid equilibrium with one another this has the effect of reducing the signal observed from the free protons. The magnitude of this effect, the magnetisation transfer ratio (MTR), is therefore an indirect indicator of the amount of macromolecular structure present per unit volume. ${ }^{24}$ The 
MTR is greater in white matter than grey matter; it is reduced within multiple sclerosis lesions, ${ }^{25-28}$ and the degree of reduction shows some correlation with disability. ${ }^{26}$ Experimental models suggest that the myelin lipid bilayer (and particularly cholesterol imbedded within it) may be a major determinant of magnetisation transfer within white matter. ${ }^{29-31}$ The MTR reduction may therefore reflect myelin loss. In Wallerian degeneration MTR is increased in the first 10 days, possibly reflecting subtle structural changes in the myelin sheath; as degeneration continues MTR falls below the baseline value. ${ }^{32}$

We have applied these MRI techniques to the optic nerve to see if we could shed further light on their pathophysiological correlates. The optic nerve is a useful model as it is commonly involved in multiple sclerosis (optic neuritis) and subserves a single class of functions which can readily be measured. Conduction can be assessed with the pattern reversal visual evoked potential (VEP), which is often abnormal in multiple sclerosis. ${ }^{33}$ The persisting delay after an episode of optic neuritis is compatible with residual demyelination within the optic nerve. ${ }^{34}$

\section{Materials and methods}

PATIENTS

Twenty patients (11 women and nine men) aged 24 to 44 (mean 33) were studied, with their written, informed consent. In all cases history was taken and examination performed by one of us (JT). Patients were classified according to the Poser criteria for the diagnosis of multiple sclerosis. ${ }^{35}$ Optic neuritis was diagnosed from the criteria of Compston et al..$^{36}$ Corrected distance and near visual acuity were recorded using Snellen chart and standard reading test types respectively. Colour vision was measured with Ishihara plates, the number of plates correctly identified (out of 17) being recorded.

\section{VISUAL EVOKED POTENTIALS}

Pattern reversal VEPs were recorded to whole field ( $26^{\circ}$ horizontally, $20^{\circ}$ vertically) and central field $\left(10^{\circ}\right.$ diameter $)$ stimulation, the stimulus being presented on a computer monitor. The amplitude and latency of the P100 were measured from an average of 200 responses. P100 latency, or interocular latency difference, was considered abnormal if it was more than 2.5 SDs above the mean of age matched controls. Amplitude was considered abnormal if it was less than $50 \%$ of that for the other eye.

\section{CONTROLS}

Six controls aged 26 to 38 (mean 31), four men and two women, consented to undergo MRI. Although none had a history of ocular disease (other than refractive error) or neurological disturbance suggestive of demyelination, they did not undergo neurological examination or VEP recording.
MAGNETIC RESONANCE IMAGING

All studies were performed on a $1.5 \mathrm{~T}$ imager (Signa, IGE Medical Systems, Milwaukee, Wisconsin) using local phased array receiver coils. In all patients, coronal fat suppressed T2 weighted fast spin echo (FSE) images were first obtained (repetition time (TR) 3400 ms, effective echo time (TE) $69 \mathrm{~ms}$, echo train length $16,512 \times 512$ or $256 \times 256$ matrix, $20 \mathrm{~cm}$ field of view, $3 \mathrm{~mm}$ contiguous interleaved slices).

For both $\mathrm{tMDC}$ and MTR measurements one slice was acquired through the intraorbital portion of each nerve. Localising images were obtained such that the images for tMDC and MTR analysis could be prescribed strictly perpendicular to the plane of the nerves, passing through the lesion (where visible on the T2 weighted images) on the affected side and through the equivalent part of the unaffected nerve. Preliminary studies in controls showed that extremely variable results were obtained from very anteriorly situated slices (within about $5 \mathrm{~mm}$ of the globe), probably due to partial volume effects from the eyeball and movement of the optic nerve (data not shown) and these were therefore avoided. Slices of 3 $\mathrm{mm}$ were acquired with a $16 \mathrm{~cm}$ field of view and $256 \times 256$ matrix, giving in plane resolution of $0.6 \mathrm{~mm}^{2}$. For the tMDC analysis a 16 echo spin echo sequence was used with a TR of $1500 \mathrm{~ms}$, echo spacing $40 \mathrm{~ms}$, and one excitation. The magnetisation transfer sequence was a non-interleaved gradient echo, TR $180 \mathrm{~ms}$, TE $8 \mathrm{~ms}$, flip angle $40^{\circ}$, acquired both with and without a $64 \mathrm{~ms}$ saturation pulse (a four lobed Hanning windowed sinc pulse giving an effective band width of about $78 \mathrm{~Hz}$ ) with an amplitude of $14.5 \mu \mathrm{T}$ at an offset of $1000 \mathrm{~Hz}$. The total MRI examination time was about one hour.

All the T2 weighted images were reviewed by one of us (IM) without reference to the clinical findings. Lesions were defined as areas of high signal within the optic nerve; the number of image slices on which they were visible permitted a measure of their length. The tMDC analysis and measurement of MTR was performed by another (JT), once again without knowledge of the clinical results. The tMDCs were fitted in turn to monoexponential, biexponential, and triexponential functions, and MTR measured from small regions of interest within the optic nerves, as detailed in the appendix.

An agarose gel phantom (Eurospin II, Diagnostic Sonar Ltd, T2 at $37^{\circ} \mathrm{C}=81 \mathrm{~ms}$ ) was also imaged to assess the accuracy of T2 measurements.

\section{STATISTICAL ANALYSIS}

Statistical analysis was carried out using Spearman's rank correlation, and MannWhitney $U$, Kruskal-Wallis, and Fisher's exact tests as appropriate. For the purposes of correlation, all measures of vision were ranked such that the worse the vision the higher the rank (a positive correlation implies correlation with increasingly poor vision). 


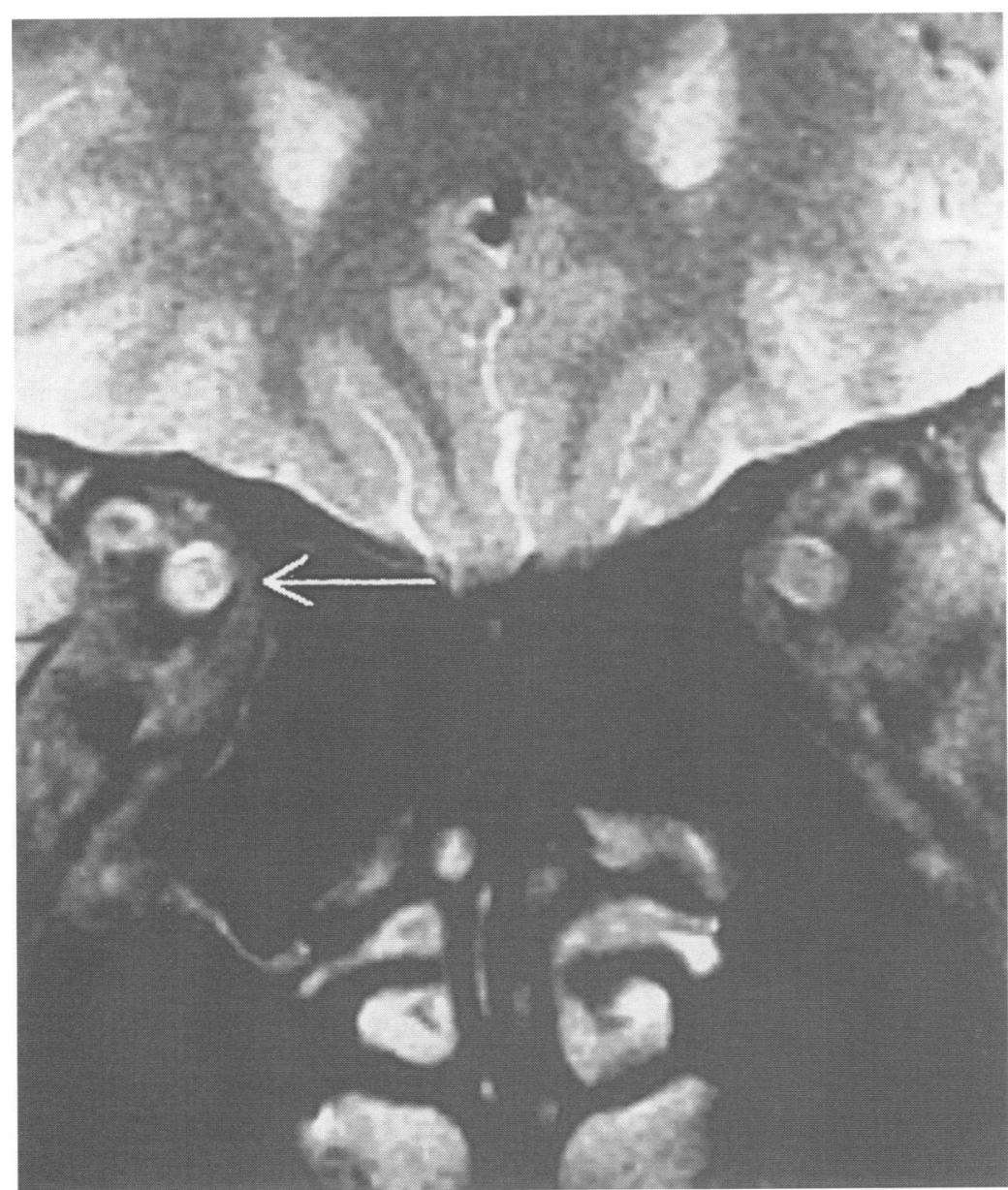

Figure 1 T2 weighted coronal FSE (FSE ${ }_{3400 / 69}, 512 \times 512$ matrix) through the optic nerves of a patient who had right optic neuritis 1 year previously. There is high signal within the right optic nerve (arrowed).
Figure 2 tMDC of the optic nerve of a patient with optic neuritis. The data are better fit by a biexponential (solid line) than a monoexponential function (dotted line). A triexponential function did not significantly improve the fit. $T 2$ is $118 \mathrm{~ms}$.

\section{Results}

Five patients had clinically definite multiple sclerosis, whereas in 15 the optic nerves were the only clinically affected site. One patient, with clinically definite multiple sclerosis, had bilateral progressive visual failure; two had progressive unilateral visual loss with a previous episode of contralateral optic neuritis. The remaining 17 had had attacks of optic neuritis between three months and 16 years earlier: three patients had had bilateral attacks. Vision in the affected eyes varied from $6 / 4$, N4.5, with normal colour vision to no perception of light.

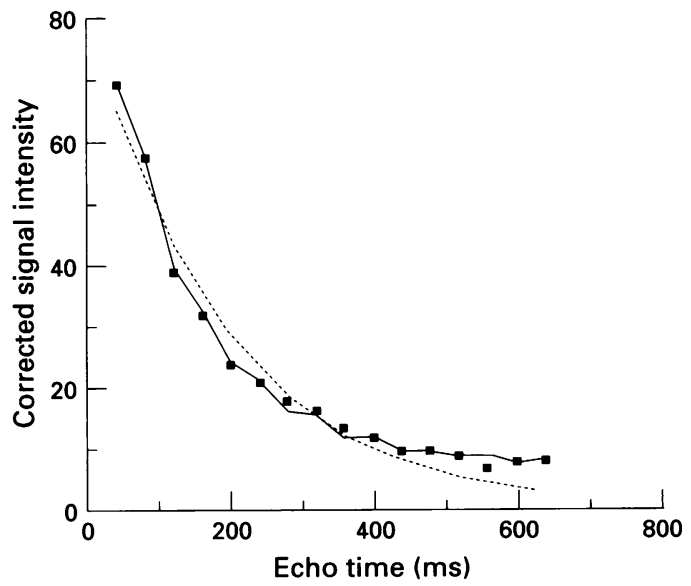

The VEPs were abnormal in 23 of the 26 clinically affected nerves, with mean whole field latency of 131 (SD) $33 \mathrm{~ms}$. One abnormal VEP was also recorded from the 14 clinically normal eyes. There were, therefore, a total of 27 optic nerves with clinical or electrophysiological evidence of previous pathological involvement ("affected" nerves). Whole field responses could not be obtained in four cases, central field responses in 10: this was generally from the eyes with the poorest vision.

In 25 of 26 clinically involved optic nerves T2 weighted FSE showed high signal within the nerve (fig 1). There was also high signal seen in two asymptomatic nerves, both of which had normal VEPs. Lesion length varied from $6 \mathrm{~mm}$ to $39 \mathrm{~mm}$ (two to 13 slices). All lesions involved the intraorbital portion of the optic nerve. Nine lesions were purely intraorbital, of which four extended as far as the front of the optic canal; 18 extended into the optic canal, of which five also involved the intracranial portion of the nerve. There was no significant relation between involvement of the nerve in the optic canal and measures of visual function (Kruskal-Wallis, $P>0.3$ ) and although there was a tendency for those with the most anterior lesions to have better vision (acuity better than 6/9) this did not reach statistical significance $(P=0.09$, Fisher's exact test). There was, however, a significant correlation between visual function (near and distance acuity, colour vision) and lesion length (see later).

In all cases the tMDCs fitted a biexponential function better than a monoexponential. In none did the triexponential function significantly improve the fit (fig 2). One control was studied six times, giving a total of 11 measurements of T2 and 10 of MTR; mean values (SD) were $74(5) \mathrm{ms}$ and 50(3) percentage units (pu) respectively. The T2 of a gel phantom was measured at $76 \mathrm{~ms}$.

There were significant differences in T2 and MTR between affected nerves and both unaffected nerves and controls (table 1). In the group of 27 affected nerves there was no significant correlation between either $\mathrm{T} 2$ or MTR and vision (near and distance acuity, colour vision). The length of the lesion, on the other hand, correlated well with visual deficit as well as with VEP amplitude,T2, and MTR (table 2). The MTR showed a strong correlation with VEP latency $(r=-0.554$, fig 3$)$. T2 did not show significant correlation with any of the measured VEP variables; it did correlate weakly with MTR $(r=-0.413$, $\mathbf{P}<0.05$ )

Table $1 T 2$ and MTR in patients and controls

\begin{tabular}{llll}
\hline & & $\begin{array}{l}\text { T2(ms) } \\
(\text { mean }(S D))\end{array}$ & $\begin{array}{l}\text { MTR (pu) } \\
(\text { mean }(S D))\end{array}$ \\
\hline Controls & $(\mathrm{n}=12)$ & $74(4)$ & $49(3)$ \\
Unaffected & $(\mathrm{n}=13)$ & $75(5)$ & $48(5)$ \\
Affected $^{\star}$ & $(\mathrm{n}=27)$ & $90(14)$ & $42(5)$
\end{tabular}

* "Affected" refers to all nerves with either electrophysiological or clinical evidence of involvement. Both T2 and MTR of affected nerves are significantly different from controls and unaffected nerves $(P<0.005$, Mann-Whitney) 
Table 2 Correlation between clinical, $M R I$, and VEP parameters.

\begin{tabular}{|c|c|c|c|c|c|}
\hline & $\begin{array}{l}\text { Whole field } \\
\text { VEP amplitude }\end{array}$ & $\begin{array}{l}\text { Whole field } \\
\text { VEP latency }\end{array}$ & $\begin{array}{l}\text { Central field } \\
\text { VEP amplitude }\end{array}$ & $\begin{array}{l}\text { Central field } \\
\text { VEP latency }\end{array}$ & $\begin{array}{l}\text { Lesion } \\
\text { length }\end{array}$ \\
\hline $\begin{array}{l}\text { Acuity } \\
\text { MTR } \\
\text { T2 } \\
\text { Lesion } \\
\quad \text { length }\end{array}$ & $\begin{array}{l}+ \text { ve, } p<0.001 \\
\text { NS } \\
\text { NS } \\
- \text { ve, } p<0.02\end{array}$ & $\begin{array}{l}\text { NS } \\
- \text { ve, } p<0.01 \\
\text { NS } \\
\text { NS }\end{array}$ & $\begin{array}{l}0.05 \\
\text { NS } \\
\text { NS } \\
- \text { ve, } p<0.05\end{array}$ & $\begin{array}{l}\text { NS } \\
- \text { ve, p<0.05 } \\
\text { NS } \\
\text { NS }\end{array}$ & $\begin{array}{l}\text { +ve, } \mathrm{p}<0.02 \\
\text { - ve, } \mathrm{p}<0.05 \\
+\mathrm{ve}, \mathrm{p}<0.05 \\
\text { - }\end{array}$ \\
\hline
\end{tabular}

$-\mathrm{ve}=$ Negative correlation; +ve $=$ positive correlation, $\mathrm{NS}=$ not significant $(\mathrm{P}>0.05)$. * Only the results for distance acuity are shown; the results for near acuity and colour vision were similar.
Figure 3 Correlation between MTR and whole field VEP latency in the affected optic nerves. (Only 23 data points are shown as in four cases no consistent VEP was recordable.)

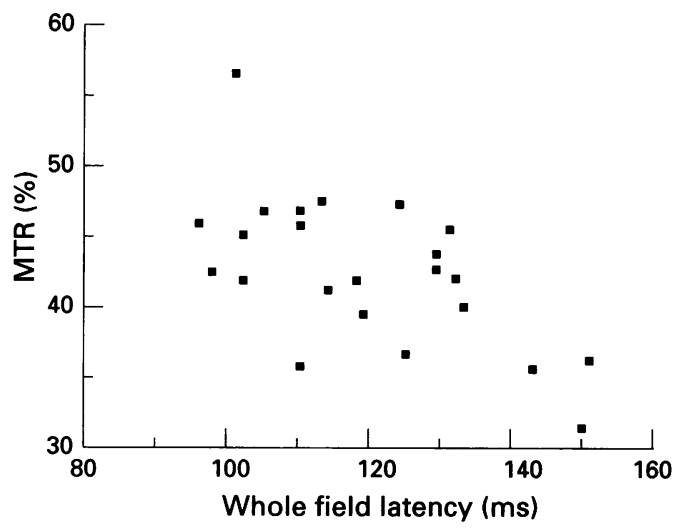

Discussion

Application of MTR and tMDC analysis to the optic nerve in vivo has not previously been reported. We experienced a number of technical difficulties in implementation. The small diameter and mobility of the nerve and its proximity to the bones of the orbit and optic canal make it difficult to obtain high resolution, high signal to noise ratio (SNR) images within a clinically acceptable time period. Without local multiarray surface coils, which produce higher SNR in the region of the optic nerve than conventional quadrature head coils, it is doubtful whether the study could have been performed. The multiecho images used to generate tMDCs in particular produced low SNR images requiring image processing to correct for the effects of noise. The resultant tMDCs always had a significantly better fit with biexponential than monoexponential functions. This is expected, as we could not completely exclude CSF from the regions of interest. Adding a third component to the fit was not justified as the biexponential fits were as good as could be expected given the noise within the data. It is possible, therefore, that there were distinct T2 components within the nerve that we were unable to resolve. Nevertheless, the multiecho sequence did allow us to obtain a measure of $\mathrm{T} 2$ from within the nerve without the confounding effects of CSF. The results obtained from scanning a phantom of known T2 suggest that the measurements were accurate; repeat studies in one control confirmed their precision.
One disadvantage of local surface coils is that signal falls rapidly with increasing distance from the coil with resultant pronounced signal non-uniformity within the image. Although methods exist for correcting this, ${ }^{37}$ they are not as useful in the optic nerves as in the spinal cord; in the first the precise position of the two coils relative to one another can vary, whereas in the second the whole array is fixed, resulting in better non-uniformity correction. This makes the assessment of high signal within the optic nerves more difficult and may have caused the apparent high signal on the T2 weighted images in two cases in whom the clinical and neurophysiological examinations were normal. Nevertheless, the sensitivity of MRI for detecting demyelinating optic nerve lesions now seems to be equivalent to that of VEPs.

Despite the limitations of the MRI methodology some interesting results have emerged. As we found previously, ${ }^{38}$ lesion length correlated with visual deficit. This result is in keeping with the pathological study of Ulrich and Groebke-Lorenz, ${ }^{19}$ who found correlation between the extent of demyelination in the optic nerves postmortem and the last recorded antemortem visual acuity. They did not undertake axon counts, so it is impossible to say whether demyelination itself or concomitant axonal loss was the main determinant of visual dysfunction. To our knowledge, no study has quantified optic nerve axonal loss in optic neuritis, nor correlated it with vision. Examination of the retinal nerve fibre layer at the optic disc gives an indirect measure of axonal loss although more than $50 \%$ of neural tissue in a given area must be lost before a defect is visible. ${ }^{39} \mathrm{MacFadyen}$ et al ${ }^{40}$ found defects in retinal nerve fibre layers in 50/114 eyes from 57 patients with definite, probable, or possible multiple sclerosis suggesting that extensive axonal loss is common; it is not possible to tell from their data whether there was any correlation with visual acuity, although seven of eight eyes with acuity of $6 / 12$ or worse had defects in retinal nerve fibre layers.

We found both T2 and MTR to be abnormal after an episode of optic neuritis. There was, however, no correlation of either with vision. This could be taken as evidence that neither is pathologically specific; equally they could be specific for pathology that does not affect those aspects of visual function measured in this study. T2 is prolonged in a variety of pathologies in the brain ${ }^{22} 41$ and is unlikely to be specific; the relation between MTR reduction and pathology remains uncertain. Although one might expect MTR reduction where there is loss of structure through either extensive axonal degeneration or myelin loss, some authors ${ }^{26} 283242$ have suggested that it principally reflects the second. Tomiak et $a l^{27}$ speculated that their finding of greater reduction in MTR in recent plaques (under a year old) than older ones signified a more pronounced effect on MTR of demyelination (and oedema) than gliosis. In this regard the finding that MTR reduction corre- 
lated with prolongation of the VEP latency is of interest. The origin of the prolongation of VEP latency in optic neuritis is complex and incompletely understood, but it is likely that slow conduction in demyelinated axons contributes. ${ }^{43}$ Axonal loss, on the other hand, does not cause substantial delays in the VEP with a well preserved wave form. The correlation with VEP latency, therefore, supports the view that MTR reduction at least in part reflects myelin loss. However, the results of Beaulieu and Allen, ${ }^{44}$ who found the MTR of unmyelinated garfish olfactory nerve to be essentially identical to that of myelinated trigeminal nerves, suggest that this may be an oversimplification. Furthermore, we only measured MTR from one $3 \mathrm{~mm}$ slice through the optic nerve, whereas the longitudinal extent of demyelination might be expected to correlate more strongly with VEP latency delay. Any conclusions must therefore remain speculative until the pathophysiological basis of MT contrast, and in particular the relative contributions of myelin and axonal loss, has been firmly established from experimental models.

The work of the NMR Research Unit is made possible by a generous grant from the Multiple Sclerosis Society of Great Britain and Northern Ireland. JT is supported by the Medical Research Council. The multiarray coils were kindly provided Research Council. The multiarray coils were kindly provided
by GE Medical Systems. We thank the physicians and surby GE Medical Systems. We thank the physicians and sur-
geons of the National Hospital, Queen Square and Moorfields geons of the National Hospital, Queen Square and Moorfields
Eye Hospital for allowing us to study patients under their care.

1 Young IR, Hall AS, Pallis CA, Legg NJ, Bydder GM, Steiner RE. Nuclear magnetic resonance imaging of the brain in multiple sclerosis. Lancet 1981;ii:1063-6.

2 Lukes SA, Crooks LE, Aminoff MJ, et al. Nuclear magnetic resonance imaging in multiple sclerosis. Ann Neurol 1983;13:567-72

3 Runge VM, Price AC, Kirshner HS, et al. Magnetic resonance imaging of multiple sclerosis: a study of pulsetechnique efficiency. AfR Am $\mathcal{f}$ Roentgenol 1984;143: 1015-26.

4 Jackson JA, Leake DR, Schneiders NJ, et al. Magnetic resonance imaging in multiple sclerosis: results in 32 cases. AfNR Am भ Neuroradiol 1985;6:171-6.

5 Sheldon J, Siddharthan R, Tobias J, et al. MR imaging of multiple sclerosis: comparison with clinical and CT examinations in 74 patients. AfR Am $f$ Roentgenol 1985; 145:957-64.

6 Scotti G, Scialfa G, Biondi A, et al. Magnetic resonance in multiple sclerosis. Neuroradiology 1986;28:319-23.

7 Ormerod IEC, Miller DH, McDonald WI, et al. The role of magnetic resonance imaging in the assessment of multiple sclerosis and isolated neurological lesions: a quantitative study. Brain 1987;110:1579-616.

8 Kappos L, Städt D, Ratzka M, et al. Magnetic resonance imaging in the evaluation of treatment in multiple sclerosis. Neuroradiology 1988;30:299-302.

9 Miller DH, Barkhof F, Berry I, Kappos L, Scotti G, Thompson AJ. Magnetic resonance imaging in monitoring the treatment of multiple sclerosis: concerted action guidelines. I Neurol Neurosurg Psychiatry 1991;54: guidelines.

10 Paty DW, Li DKB, the UBC Multiple sclerosis/MRI Study Group, the IFNB Multiple Sclerosis Study Group. Group, the IFNB Multiple Sclerosis Study Group. Interferon beta-1b is effective in relapsing-remitting multiple sclerosis. II. MRI analysis results of a multicenter, randomized, double-bir

$11 \mathrm{Li} \mathrm{DKB}$, Mayo J, Fache S, et al. Lack of correlation between clinical manifestations and lesions of multiple sclerosis as seen by NMR [abstract]. Neurology 1984;34 (suppl 1):136.

12 Jacobs L, Kinkel WR, Polachini I, Kinkel RP. Correlations of nuclear magnetic resonance imaging, computerized tomography and clinical profiles in multiple sclerosis. Neurology 1986;36:27-34.

13 Stewart JM, Houser OW, Baker HL, O'Brien PC, Rodriguez M. Magnetic resonance imaging and clinical relationships in multiple sclerosis. Mayo Clin Proc 1987; 62:174-84.

14 Huber SJ, Paulson GW, Chakeres D, et al. Magnetic resonance imaging in multiple sclerosis: Comparison with clinical, CSF and visual evoked potential findings. AfNR Am $₹$ neuroradiol 1988;9:59-67.

15 McDonald WI, Miller DH, Barnes D. The pathological evolution of multiple sclerosis. Neuropath Applied Neurobiol 1992;18:319-24.
16 Namerow NS, Thompson LR. Plaques, symptoms, and the remitting course of multiple sclerosis. Neurology 1969;19:765-4.

17 Ghatak NR Hirano A, Lijtmaer $\mathrm{H}$, Zimmerman $\mathrm{HM}$ Asymptomatic demyelinated plaque in the spinal cord. Arch Neurol 1974;30:484-6.

18 Wisniewski HM, Oppenheimer D, McDonald WI Relation between myelination and function [abstract]. $\mathcal{F}$ Neuropath Exp Neurol 1976;35:327.

19 Ulrich J, Groebke-Lorenz W. The optic nerve in multiple sclerosis. A morphological study with retrospective clinico-pathological correlations. Neuro-ophthalmology 1983; 3(part 3):149-59.

20 McDonald WI, Sears TA. The effects of experimental demyelination on conduction in the central nervous system. Brain 1970;93:583-98.

21 Larsson HBW, Frederiksen J, Kjær L, Henriksen $O$, Olesen $J$. In vivo determination of $T_{1}$ and $T_{2}$ in the brain of patients with severe but stable multiple sclerosis. Magn Reson Med 1988;7:43-55.

22 Barnes D, Munro PMG, Youl BD, Prineas JW, McDonald WI. The longstanding MS lesion. A quantitative MRI and electron microscopy study. Brain 1991 114:1271-80.

23 Armspach J-P, Gounot D, Rumbach L, Chambron J. In vivo determination of multiexponential $T_{2}$ relaxation in the brain of patients with multiple sclerosis. Magn Reson Imag 1991;9:107-13.

24 Wolff SD, Balaban RS. Magnetisation transfer contrast MTC) and tissue water proton relaxation in vivo. Magn Reson Med 1989;10:135-44.

25 Dousset V, Grossman RI, Ramer KN, et al. Experimenta allergic encephalomyelitis and multiple sclerosis: Lesion characterization with magnetization transfer imaging. Characterization with magn

26 Gass A, Barker GJ, Kidd D, et al. Correlation of magnetisation transfer ratio with clinical disability in multiple sclerosis. Ann Neurol 1994;36:62-7.

27 Tomiak MM, Rosenblum JD, Prager JM, Metz CE. Magnetization transfer: a potential method to determine the age of multiple sclerosis lesions. AfNR $A m f$ Neuroradiol 1994;15:1508-69.

28 Hiehle JF, Grossman RI, Ramer KN, Gonzales-Scarano F Cohen JA. Magnetization transfer effects in MRdetected multiple sclerosis lesions: comparison with gadolinium-enhanced spin-echo images and nonenhanced $T_{1}$-weighted images. $A \mathcal{F} N R$ Am $\mathcal{F}$ Neuroradiol 1995;16:69-77.

29 Koenig SH, Brown RD, Spiller M, Lundbom $N$ Relaxometry of brain: why white matter appears bright Relaxometry of brain: why white matter a
in MRI. Magn Reson Med 1990;14:482-95.

30 Fralix TA, Ceckler TL, Wolff SD, Simon SA, Balaban RS Lipid bilayer and water proton magnetization transfer effect of cholesterol. Magn Reson Med 1991;18:214-23.

31 Koenig SH. Cholesterol of myelin is the major determinant of grey-white contrast in MRI of brain. Magn Reson Med 1991;20:285-91.

32 Lexa FJ, Grossman RI, Rosenquist AC. MR of wallerian degeneration in the feline visual system: characterisation by magnetization transfer rate with histopathological correlation. AfNR Am $\Im$ Neuroradiol 1994;15:201-12.

33 Halliday AM, McDonald WI, Mushin J. Visual evoked response in diagnosis of multiple sclerosis. BMF 1973; 4:661-4.

34 McDonald WI. Pathophysiology of conduction in central nerve fibres. In: Desmedt JE, ed. Visual evoked potentials in nerve fibres. In: Desmedt JE, ed. Visual evoked potentials in man: nerw

35 Poser CM, Paty DW, Scheinberg L, et al. New diagnostic criteria for multiple sclerosis: guidelines for research protocols. Ann Neurol 1983;13:227-31.

36 Compston DAS, Batchelor JR, Earl CJ, McDonald WI Factors influencing the risk of multiple sclerosis developing in patients with optic neuritis Brain 1978;108: 241-62.

37 Tofts PS, Barker GJ, Simmons AMK, et al. Correction of nonuniformity in images of the spine and optic nerve from fixed receive-only surface coils at 1.5T. F Comp Assist Tomogr 1994;18:997-1003

38 Miller DH, Newton MR, Van der Poel JC, et al. Magnetic resonance imaging of the optic nerve in optic neuritis. resonance imaging of the
Neurology 1988;38:175-9.

39 Quigley HA, Addicks EM. Quantitative studies of retinal nerve fiber layer bundles and bundle defects. Arch nerve fiber layer bundles and
Ophthalmol 1982;100:807-14.

40 MacFadyen DJ, Drance SM, Douglas GR, Airaksinin PJ, Mawson DK, Paty DW. The retinal fiber layer, neuroretinal rim area, and visual evoked potentials in MS Neurology 1988;38:1353-8.

41 Larsson HBW, Frederiksen J, Petersen J, et al. Assessmen of demyelination, edema, and gliosis by in vivo determination of $\mathrm{T} 1$ and $\mathrm{T} 2$ in the brain of patients with acute attacks of multiple sclerosis. Magn Reson Med 1989;11: 337-48

42 Dousset V, Brochet B, Vital A, Nagem F, Bonnet J, Caillé JM. MR imaging including diffusion and magnetization transfer of chronic relapsing experimental encephalomyelitis - correlation with immunological and pathological data [abstract]. Proceedings of the Society of Magnetic Resonance, San Francisco 1994;1401.
Ral data [abstract]. Proceedings of the

43 McDonald WI. The pathophysiology of multiple sclerosis. In: McDonald WI, Silberberg DH, eds. Multiple sclerosis Butterworths, 1986:114-26.

44 Beaulieu C, Allen PS. Some magnetisation transfer properties of water in myelinated and nonmyelinated nerves 

[abstract]. Proceedings of the Society of Magnetic

45 Miller AJ, Joseph PM. The use of power images to perform quantitative analysis on low SNR MR images. Magn Reson Imaging 1993;11:1051-6.

\section{Appendix: tMDC analysis and MTR measurement}

TMDC ANALYSIS

Quantitative analyses were performed on a Sun Workstation using image analysis software (Dispimage, developed by one of us (DLP) and Viewit, CS Potter and PJ Moran, National Centre for Supercomputing Applications, University of Illinois) and a graph plotting package (Xvgr, P Turner, Oregon Graduate Institute of Science and Technology).

The multiecho images were first corrected using a variation on the method of Miller and Joseph ${ }^{45}$ : a result of the magnitude reconstruction method used to generate conventional images is that in situations of low signal to noise ratio (SNR), such as on later echoes of the multiecho sequence, the mean signal intensity of noise is positive, rather than zero.

This results in signal intensities at long echo times that are artefactually raised and this influences measurements of T2. Miller and Joseph showed that a measure of signal intensity, unbiased by the effects of noise, can be derived where:

$$
\begin{aligned}
& \qquad \boldsymbol{M}_{c}^{2}=\boldsymbol{M}^{2}-\boldsymbol{M}_{0}^{2} \\
& \boldsymbol{M}_{c}=\text { corrected signal intensity } \\
& \boldsymbol{M}=\text { uncorrected signal intensity } \\
& \boldsymbol{M}_{0}=\text { mean background signal intensity (air noise) }
\end{aligned}
$$

We therefore squared the signal intensity of the image on a pixel by pixel basis and then subtracted the square of the mean background intensity, taken from a large region of interest placed in air. We then placed regions of interest on the corrected images within the optic nerves to generate decay curves; the corrected tMDCs were then derived by taking the square root of these curves. A non-linear curve fitting algorithm (within Xvgr) was used to fit the tMDCs in turn to monoexponential, biexponential, and triexponential functions. For biexponential and triexponential functions, one component was assumed to be CSF within the nerve sheath and for this component the T2 was fixed at $2100 \mathrm{~ms}$. This value had been measured empirically in 15 subjects from 30 regions containing CSF in the frontal subarachnoid space.
MONOEXPONENTIAL FUNCTION

$$
y=k \cdot\left(e^{-T H / T 2}+B \cdot[\bmod ]\right)
$$

BIOEXPONENTIAL FUNCTION

$$
y=k \cdot\left(A e^{-\frac{T E}{T 2}}+(1-A) e^{-\frac{T E}{2100}}+B \cdot[\bmod ]\right)
$$

TRIEXPONENTIAL FUNCTION

$$
\begin{gathered}
y=k \cdot\left(A^{\text {shon }} e^{\frac{-T E}{T 2^{\text {thon }}}}+A^{\text {long }} e^{\frac{-T E}{T 2^{\text {long }}}}\right. \\
\left.+\left(1-\left(A^{\text {shorn }}+A^{\text {long }}\right)\right) e^{\frac{-T E}{2100}}+B \cdot[\bmod ]\right)
\end{gathered}
$$

where $T 2, T 2^{\text {shon }}$ and $T 2^{\text {long }}$ are the $T 2$ values and $A$, $A^{\text {shor }}$ and $A^{\text {long }}$ the proportions of the various components, and $\boldsymbol{k}$ is an overall scaling constant. [mod] is a term which allows for inaccuracies in the $180^{\circ}$ pulses which results in lower than expected intensities on the odd numbered echoes, defined as:

$$
[\bmod ]=(1-[\text { modulo }(T E, 80) / 40])
$$

(modulo $(T E, 80)$ is the remainder when the echo time is divided by 80 , such that [mod] is 0 for even numbered echoes and 1 for odd numbered echoes.)

For a more complex function to be accepted over a more simple one it had to satisfy three condition; firstly, that it was biologically meaningful (all proportions positive, all $T 2 s$ positive and less than $5000 \mathrm{~ms}$ ); secondly, that it significantly improved the fit (modified $F$ test, $\mathrm{P}<0.05$ ) and lastly, that the more simple function did not fit the data as well as could be expected given the inherent noise within it. This was assessed by comparing (by $F$ test) the fit to that of data obtained from the agarose gel phantom.

MTR MEASUREMENT

MTR (in units of percentage units, pu) was caculated within the optic nerves from:

$$
M T R=\frac{S I^{0}-S I^{S}}{\mathrm{SI}^{0}} \times 100
$$

where $\mathrm{SI}^{\mathrm{S}}$ and $\mathrm{SI}^{0}$ are the mean signal intensities from within the optic nerves with and without the saturation pulse respectively. In all cases, a small region (4 pixels) taken from the centre of the nerve was used to minimise partial volume effects from surrounding CSF. 